\title{
Erratum: Quantized orbital-chasing liquid metal heterodimers directed by an integrated pilot-wave field [Phys. Rev. Fluids 5, 053603 (2020)]
}

\author{
Jianbo Tang $\odot$, Xi Zhao, and Jing Liu \\ Q (Received 5 August 2021; published 17 August 2021)
}

DOI: 10.1103/PhysRevFluids.6.089902

During our ongoing experiments, we noticed a calibration issue with the acceleration measurement in our system which led to acceleration amplitudes ten times higher than the actual values. Consequently, the experimentally measured driving acceleration amplitude $\gamma_{0}$ including also $\gamma_{B}$ and $\gamma_{F}$ should be multiplied by a factor of 0.1 .

This correction applies to Figs. 1, 3, 4, 5, 8, and 9.

This error does not affect the results and conclusions of the article. 